\title{
Improvement and Modelling of the Knelson Concentrator
}

\author{
Lokman Aziz AbdulKareem \\ Erbil Technical College
}

\begin{abstract}
The Knelson concentrator is a centrifugal separator which was originally and successfully designed for extracting free gold particles. Despite of its wide range of applications, the exact concentration mechanism of the device is not fully understood.

The purpose of this research was to determine the optimum parameters and improve the performance of knelson concentrator by investigating the effects of artificial porous beds and pulsed water jackets on the performance of the device.

Artificial bed consists of glass beads of range $1.5-2 \mathrm{~mm}$ diameter have been used. It was found that two techniques can improve the performance of the batch Knelson concentrator. The experimental work showed that improvement ratio of concentrator was increased about 1.55 for flow rate $8 \mathrm{l} / \mathrm{m}$ compared with no bed for the same flow rate. In addition, the improvement ratio was increased about 1.25 when pulsed water was used.

Another purpose of this work was to develop mathematical model approaches to understand the fundamental mechanism of the Knelson concentrator.

In addition, two mathematical model of the Knelson concentrator have been developed.
\end{abstract}

Key words: Kneslon Concentrator, Pulsation, Porous Bed, Mathematical Model

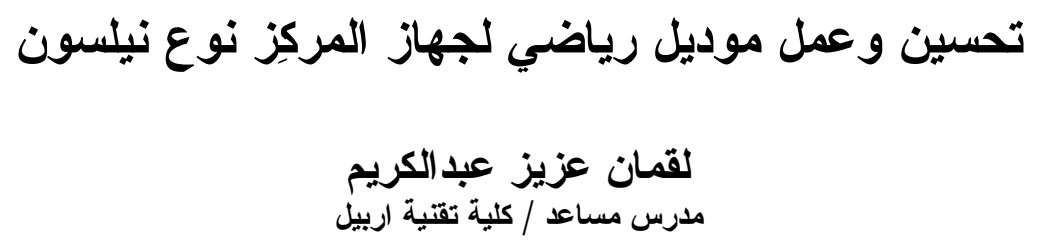

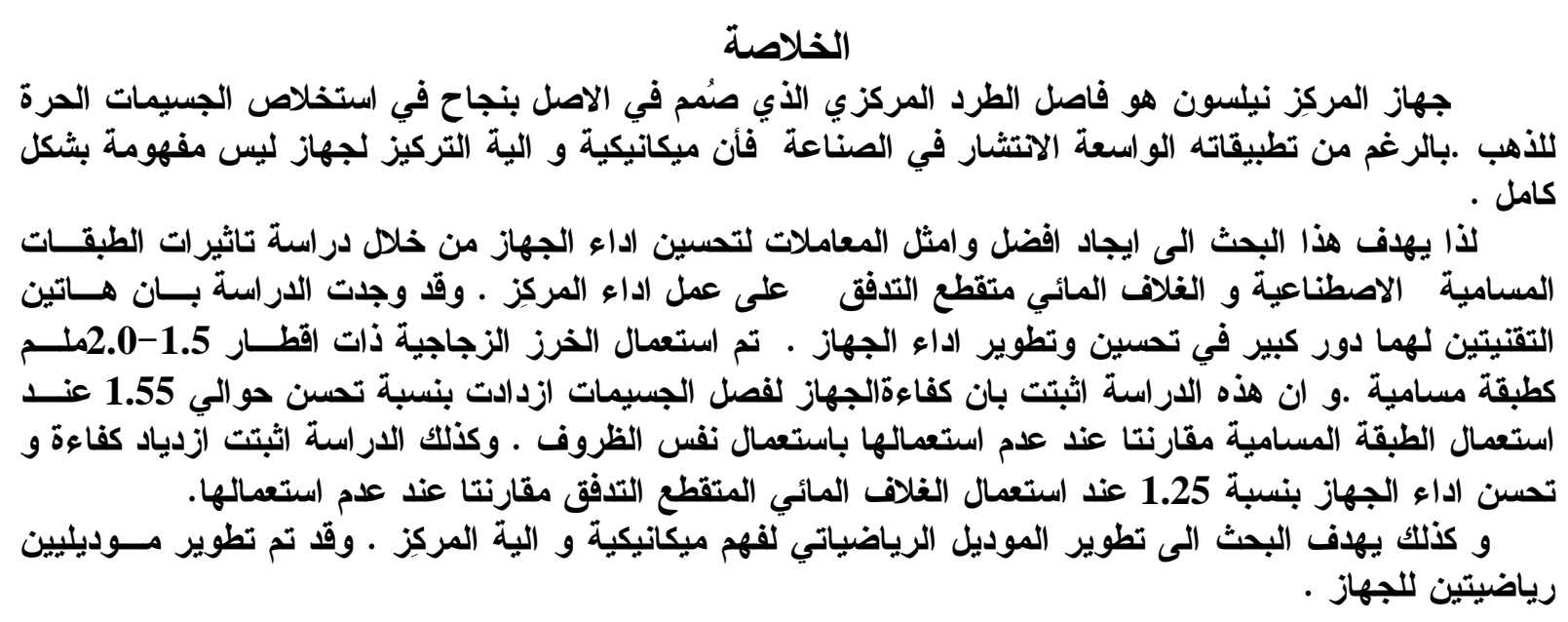




\section{Nomenclature}

$C_{D} \quad$ Drag coefficient

$\mathrm{d}_{\mathrm{p}} \quad$ particle diameter

g Gravitational acceleration

G Centrifugal acceleration

\begin{tabular}{c}
$\mathrm{m}$ \\
$\mathrm{m} / \mathrm{s}^{2}$ \\
$\mathrm{~m} / \mathrm{s}^{2}$ \\
$\rho_{f} u_{m} d_{p}$ \\
\hline$\mu(1-\varepsilon)$ \\
$\mathrm{m} / \mathrm{s}$ \\
$\mathrm{m} / \mathrm{s}$ \\
$\mathrm{m} / \mathrm{s}$ \\
$\mathrm{m} / \mathrm{s}$ \\
$\mathrm{m} / \mathrm{s}$ \\
$\mathrm{N}$ \\
$\mathrm{N}$ \\
$\mathrm{m}$
\end{tabular}

Greek letters

$\varepsilon \quad$ Porosity

$\mu$ Fluid viscosity $\quad \mathrm{Ns} / \mathrm{m}^{2}$

$\operatorname{Re}_{p} \quad$ Reynolds number

$\omega$ Angular velocity

$\mathrm{rad} / \mathrm{sec}$

$\rho_{f} \quad$ Density of fluid medium $\quad \mathrm{kg} / \mathrm{m}^{3}$

$u_{f} \quad$ Actual fluid velocity

$\rho_{h} \quad$ Density of heavy mineral. $\quad \mathrm{kg} / \mathrm{m}^{3}$

$u_{t} \quad$ Terminal velocity

$\rho_{l} \quad$ Density of light mineral.

$\mathrm{kg} / \mathrm{m}^{3}$

$\rho_{p} \quad$ Density of particle

$\mathrm{kg} / \mathrm{m}^{3}$

$u_{m} \quad$ Minimum fluidization velocity $\mathrm{m} / \mathrm{s}$

$u_{p} \quad$ Particle velocity

$F_{c} \quad$ Centrifugal force

$F_{b} \quad$ Buoyancy force

$r$ radial coordinate $\rho^{2}$

\section{Introduction}

\subsection{Continuous Knelson concentrator}

The Knelson Continuous Variable-Discharge Concentrator (CVD6) [1] shown in Fig.(1-1) was developed to address specific mineral recovery applications which are suitable to enhanced gravity separation but, due to comparatively high content of target mineral, are not practical for conventional batch concentration methods.

The Knelson concentrator CVD6 is also the only continuous-type centrifugal concentrator available that utilizes Knelson's widely proven patented fluidization technology.

The continuous-type technology differs from the batch-type technology by delivering a continuous stream of concentrate. The CVD6 will discharge the concentrate while simultaneously processing fresh feed, whereas batch-type units must be stopped intermittently to remove concentrate. Continuous-type Knelson Concentrators are suited to applications where the target mineral exceeds $0.5 \%$ of the total feed solids.

All CVD6 units are equipped with fully automated control systems to allow the concentrator to operate hands-free without disruptions. The operator interface allows for onthe-fly adjustment of operating variables such as fluidization water flow, G-force and concentrate mass yield. Fluidization of feed creates an effective separating medium and also ensures smooth operation and reduced abrasive wear. The fluidized CVD6 can effectively deslime most feeds and is not prone to the plugging problems that have plagued other types of centrifugal concentrators. 


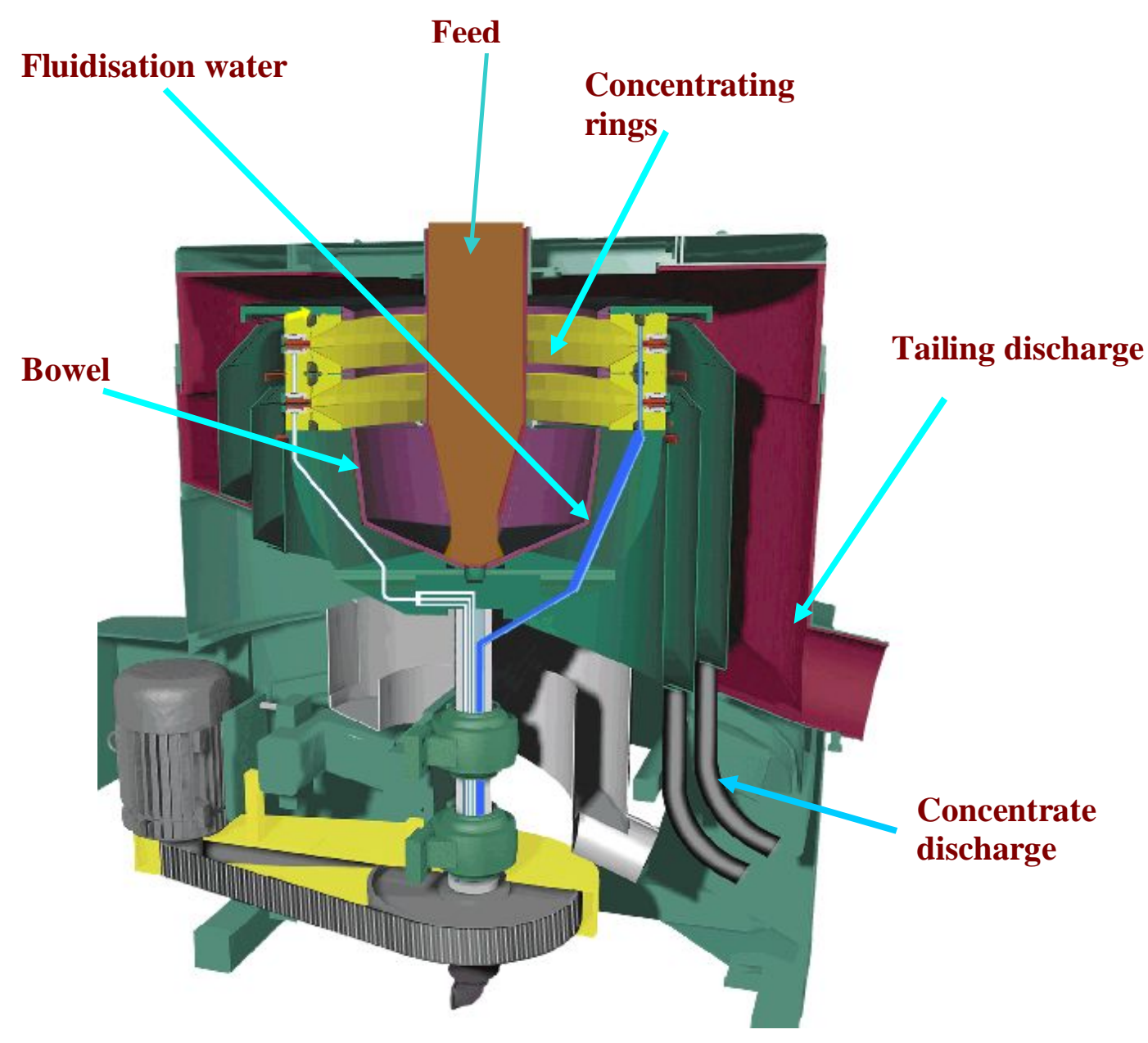

Figure 1-1. Schematic of Continuous Knelson

Concentrator (www.knelson.com)

A typical continuous-type Knelson Concentrator works like this: Water is introduced through a series of fluidization holes located in the concentrate ring(s). Feed slurry is introduced through the feed tube. When the slurry reaches the bottom of the cone it is forced outward and driven up the cone wall toward the concentrate ring(s). The slurry fills the concentrate ring(s) to create a fluidized concentrate bed. Pinch valves are actuated allowing concentrates to be drawn from the concentrate ring(s) at a controlled rate. Concentrates report to a dedicated launder while tailings flow out the top of the cone into the tailings launder [2]. The Continuous Knelson Concentrators have seen successfully used in mineral processing industry, especially for heavy minerals separation. The potential applications could be coal cleaning and solid waste separation as other gravity separators have been used [2] [3].

\subsection{Researches on Continuous Knelson Concentrators}

Early test work used synthetic mixture of magnetite and quartz on CVD6 [4]. The four main operating variables: fluidization water flow rate, bowl speed, pinch valve open time and closed time have been investigated. Mixtures of quartz and two sizes of magnetite were prepared with grades of $1 \%$ and $4 \%$ magnetite by weight. For each test the operating parameters levels were set and the CVD6 was run for at least 15 minutes to ensure steady 
state conditions prior to sampling in the feed, concentrate and tailings. The samples were filtered, oven dried and split for assay.

For all operating parameters, changing the levels to increase grade causes a reduction in recovery and vice versa. A good understanding of the effects of each variable is important to develop an optimum performance operating strategy. The general trends for operating variables are: Increasing fluidization water flow rate, bowl speed and pinch valve open time resulted in the grade decreased and recovery increased; increasing pinch valve close time resulted in grade increased and recovery decreased. Some threshold points for CVD6 were also found. Fluidization water had a little effect on grade and recovery as long as it was run above $6 \mathrm{gpm}$. Analysis of the effect of bowl speed showed that bowl speed should be run below $700 \mathrm{rpm}$.

Some novel applications have been explored as well. The National Defence Centre for Environmental Excellence (NDCEE) in USA tried to use the CVD6 to scavenge the metals that remained in pulverized printed wiring board (PWB) material after initial separation processing [5]. Approximately 80 pounds of material (particles size below 106mm) was recirculated for each operational test. A series of tests demonstrated that the Knelson CVD6 was an improvement to the PWB separation system and increased the likelihood of passing toxicity characteristic leaching procedure limits of EPA's regulations.

\subsection{Binary Solid Liquid Fluidization Separation 1.3.1 Introduction}

Fluidized beds also provide a method for particle separation based on density difference [6]. Liquid-solid fluidization of a mixture of particles is common to a variety of industrial processes, with specific application in mineral classification, elutriation, and crystallization and fluidization bed leaching [7]. When a liquid fluidized bed containing two or more particles with different nature characterized by size, density, shape and surface property, a driving force for particle separation is established [8].

For two particles differing in their size as well as density such that the larger particles are lighter and the smaller ones are denser, the segregation pattern is more complex and could lead to layer inversion [7]. The layer inversion phenomenon was first studied systematically by [9].

\subsubsection{Theory development}

Different theoretical explanations for layer inversion have been reviewed by [10]. With the exception of [11] who consider layer inversion to be the result of a mixing effect, predictions of the phenomenon are based on the experimental finding of the fluid dynamic equilibrium in the bottom layer of the bed: i.e. the total fluid-particle interaction force on each particle species should balance its weight. Some degree of success has been obtained, although none stands out as being definitely preferable to the others [12]; [13]; [14] stated that layer inversion would occur at the particular fluid velocity where the bulk densities of the two monocomponent beds are equal. This suggestion cannot explain the observed mixing segregation situation.[15] extended this idea and proposed the complete segregation model, which used a pressure drop constraint relationship. This model was support by some experimental observations [8].

[15] Model compares well with all the previous results on the inversion phenomenon. However, it is still lacking [7]. Hu developed a new model that incorporated the bulk density and voidage fluctuation to interpret the layer inversion. 
A pseudo fluid model has been developed to explain the segregation velocity [16]. It is assumed to contain the pseudo fluid, which consists of the homogeneous mixture of the fluid and smaller and denser particles. The larger particles are assumed to be present in the pseudo fluid. The properties of the pseudo fluid are used to compute the segregation velocity of the larger particles using the correlation [17].

\subsection{Research on the Separation Mechanism}

There are some researches on the concentration mechanism from the perspective of the mechanics of individual particle. The equations for determining the instantaneous velocities of a spherical solid particle in the Kneson concentrator have been tentatively derived based on Newton's second law of motion [18]. Although the solutions of the equations of motion were derived only for the Stoke's law region, their physical meaning might be applied to the particle motion in high Reynolds number flows. It can be expected from the equations of motion for a spherical particle in the radial direction that the instantaneous radial settling velocity and percolation or migration velocity of a particle moving in the inner bowl depend not only on the centrifugal acceleration, particle size and density, but also on the flow velocity. The axial velocity of a particle is a function of centrifugal acceleration, the axial velocity of the following film, and the physical characteristics of the particle. Conversely, the retention time of a particle or the height of the inner bowl may be estimated if the vertical velocity of the particle is known. As a whole, the balance between the ability of the Knelson concentrator to increase the radial settling and percolating velocity of dense particles and to decrease the retention time of gangue particles without unacceptably eroding dense particles already captured is quite a delicate process. Among the variables which might affect this balance, the centrifugal acceleration of the Knelson concentrator and appropriate fluidization of the flowing slurry are the most critical.

The mode of particle behaviour in the Knelson concentrator has also been investigated by [19]. He successfully used the equations (1.4.1, 1.4.2 and 1.4.3) for Stoles' settling velocity to calculate the cutting point diameter of magnetite in the synthetic magnetite and quartz system.

$$
\begin{aligned}
& u_{f}=\frac{u_{s}}{\varepsilon} \\
& \frac{C_{D}}{\operatorname{Re}_{p}}=\frac{4 g \mu\left(\rho_{p}-\rho_{f}\right)}{3 \rho_{f}{ }^{2} u_{f}{ }^{3}} G \\
& d=\frac{\operatorname{Re}_{p} \mu}{\rho_{f} u_{f}}
\end{aligned}
$$

Furthermore, he found that by creating an artificial porous concentrate bed in the rings of the concentrating bowl, a significant increase in the concentrate grade could be obtained. However, in general, this was at the expense of lower magnetite recoveries. From this it can be concluded that the main concentration mechanism occurs at the surface of the concentrate bed and that main factor leading to improved grades is the artificial bed occupying volume deep within the concentrate rings, which would otherwise be occupied by gangue materials.

\section{Experimental Set up}

The 7.5" pilot scale batch Knelson concentrator and the Knelson continuous variable discharge (CVD6) were used as experimental devices, coal, mixture of quarts and magnetite 
as feed materials and the glass beads $(1.5-2.5 \mathrm{~mm})$ were used to create artificial beds. The effects of varying the water jacket flow rate, artificial beds and pulsed water on the grade of the concentrate were investigated.

At first the Batch Knelson Concentrator was involved. Feed materials were made wet and homogeneous in a bucket. Secondly, it was to set up the Knelson concentrator's parameters: spin speed and water jacket flow rate. In the experiments done, it needed to keep the spin speed to make the centrifugal force at $60 \mathrm{G}$ $\left(\mathrm{m} / \mathrm{s}^{2}\right)$ because the real commercial device is running at this speed. After the device was running, the feed materials was poured into the funnel of the Knelson, if artificial beds were used, glass beads were fed before feeding synthetic materials to create artificial beds. When the feeding was finished, the device was stopped, the bowl (chamber of samples) Figure 2-1, was taken out and the concentrate was flushed out of

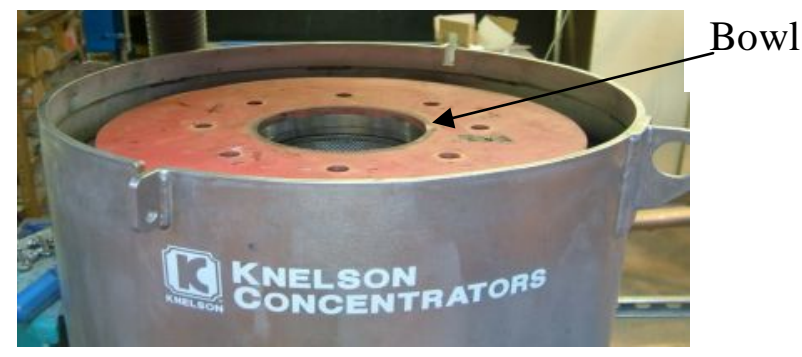

Figure 2-1. Photo of CVD6 and The bowl the rings. If there were glass beads in the concentrate, the glass beads were needed to be separated first by an appropriate size sieve. And then the concentrate was filtrated, and dried in the oven overnight. At last the concentrates were analyzed.

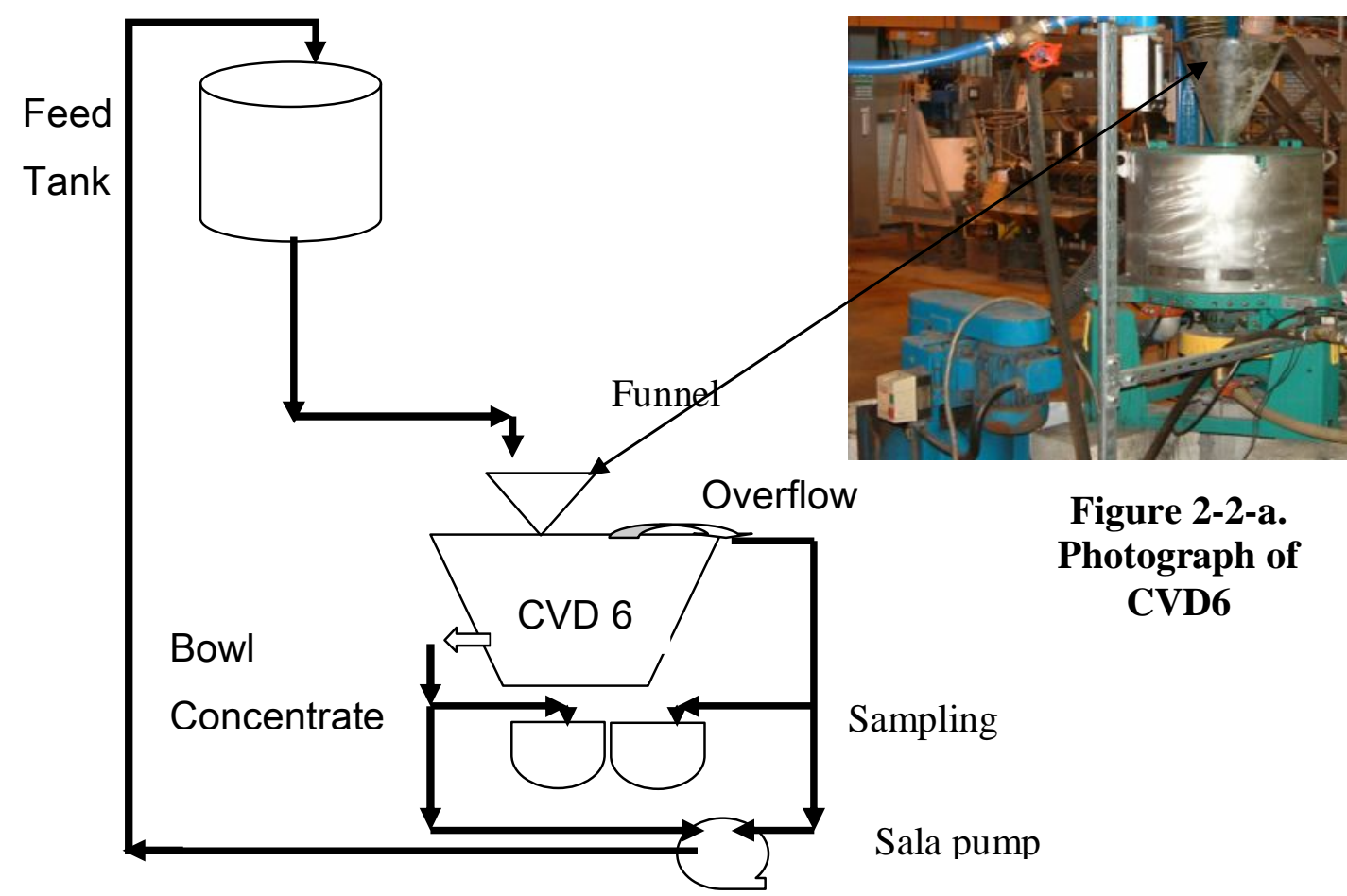

Figure 2-2-b. Layout of CVD 6 Experiments Circuit

The CVD6 experiment flowchart Figure 2-2a, b. The test facility consists of an agitated feed tank; CVD6 and a Sala pump that combines concentrate and overflow to the feed tank. Samples of each stream collected during the experiment are dried and weighted for analysis

\section{Results and Discussion}

\subsection{Feed Material}


In order to obtain good and precise results the experiments have been carried out with synthetic materials at first. A mixture of magnetite and quarts was used as feed material. The specific gravity of the magnetite was 5.18 and the specific gravity of the quarts was 2.65 Figure 3-1. The quartz was from Tilcon Industrial Minerals and particle was below 125 microns. In order to mimic fine materials, the magnetite was grinded in the ball mill. The particle size distribution was determined by wet sieving. Russell sieve was used. It can be seen in the Figure 3-2 that grade of cumulative is increasing with increasing the size of particle. Figure shows that grade of 850 micron particles is the highest percentage.

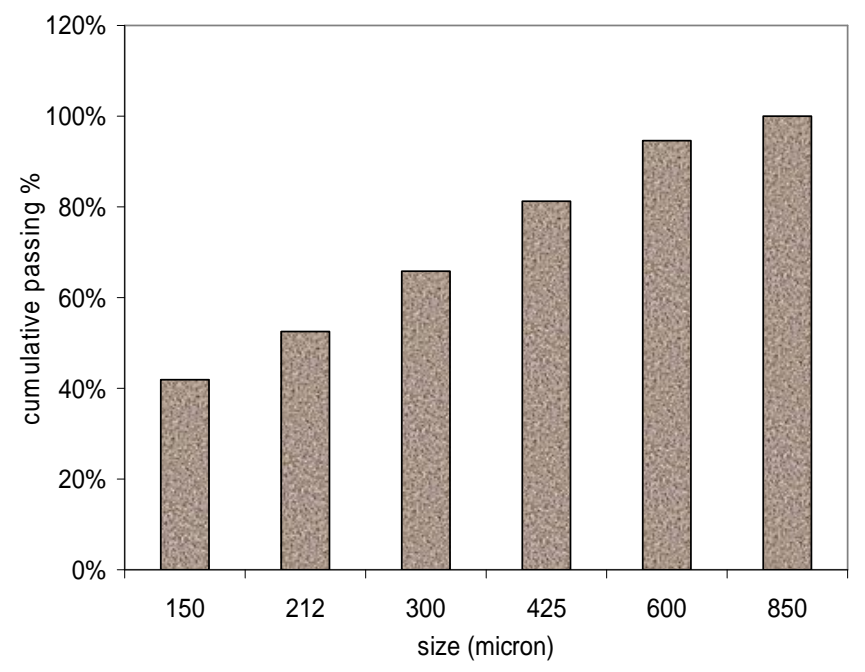

Figure 3-2. Particle Size Distributions

was grinded in the rod mill. The particle size distribution is given in Figure 3-3.

\subsection{Artificial Bed Tests}

The effect of setting up an artificial bed in the concentrate ring of the bowl was initially investigated by [19].A feed mixture of quartz and magnetite was prepared with an overall magnetite grade of $5 \%$ and then mixed with water(18 litre) at a solid concentration of $10 \%$. Each run was conducted using $2 \mathrm{~kg}$ (1900g of quartz and $100 \mathrm{~g}$ of magnetite) of feed introduced at a constant rate. The mixtures was fed into the unit at a feed rate of $250 \mathrm{~g} / \mathrm{min}$. Test runs with and without artificial beds were carried out using water flow rates of 6,8 and

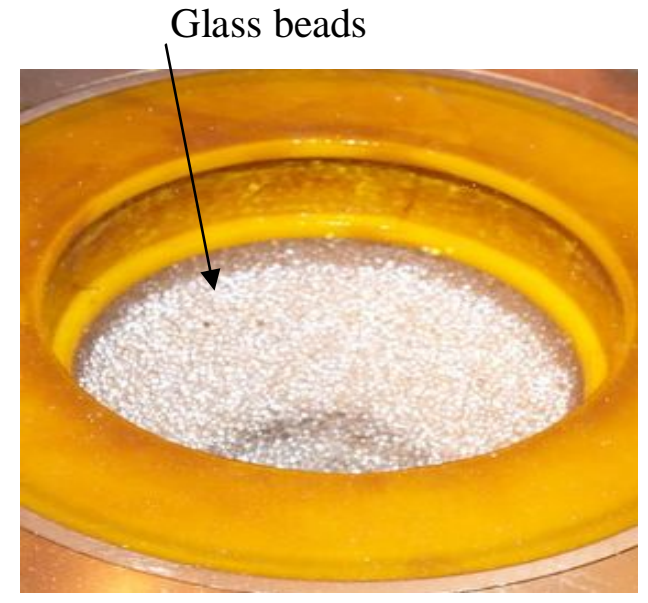

Figure 3-4. Glass beads in the bowl 
$10 \mathrm{l} / \mathrm{min}$ and a fixed centrifugal force of $60 \mathrm{G}\left(\mathrm{m} / \mathrm{s}^{2}\right)$. The artificial bed consisted of $1.5 \mathrm{~kg}$ of glass beads and of a range size of 1.5-2.0 mm was fed into the batch Knelson unit before the real sample was added. The glass beads filled the whole chamber and formed an artificial porous bed Figure 3-4. This mass was sufficient to occupy the total chamber volume. After each run, the product in the concentrating ring was flushed out of the chamber, filtered and oven dried for grade analysis.

The artificial beds occupy the volume deep within the concentrate rings, which would be occupied by gangue. The experiments were performed under the same operating conditions as no artificial beds used. The effect of increasing the water jacket flow rate on the artificial beds and no artificial beds is illustrated in figure 3-5.

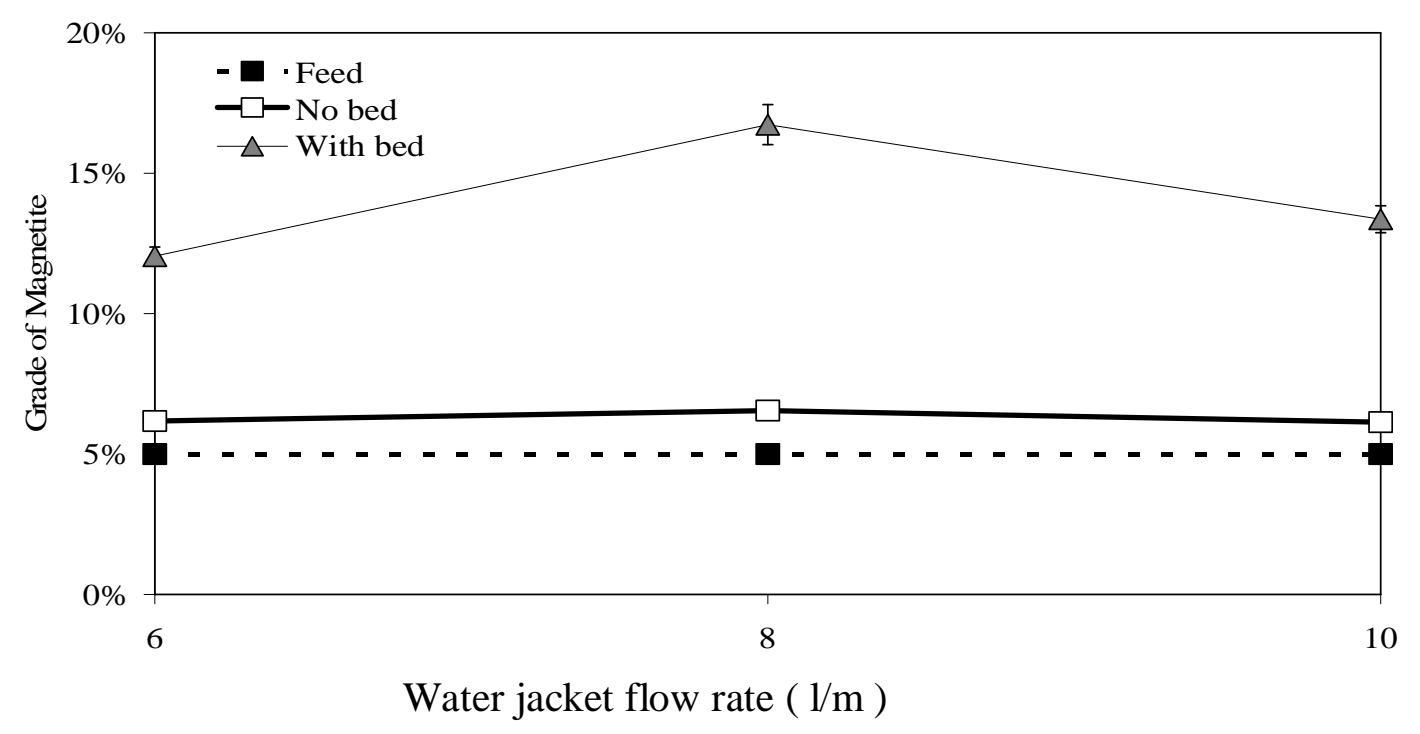

Figure 3-5.The Effect of Artificial Beds

Figure 3-5 shows the variation in the total magnetite grade with increasing water jacket flow rate for tests with and without artificial beds. The feed magnetite grade was $5 \%$. At normal operating conditions, without an artificial bed, the concentrate grades were about $6 \%$. In Table 1 the concentrate grades obtained in corresponding tests with artificial beds were nearly double, and performance of concentrator has improved about 1.55 , with the grade varying from $12 \%$ at $6 \mathrm{~L} / \mathrm{min}$ to $16 \%$ at $8 \mathrm{~L} / \mathrm{min}$.

Table 1. Ash contents with and with out bed in product

\begin{tabular}{|l|r|r|r|}
\hline Feed & $5 \%$ & $5 \%$ & $5 \%$ \\
\hline No bed & $6.17 \%$ & $6.55 \%$ & $6.14 \%$ \\
\hline With bed & $12.05 \%$ & $16.73 \%$ & $13.36 \%$ \\
\hline Flow rate & 6 & 8 & 10 \\
\hline Improvement & 0.951 & 1.5559 & 1.1773 \\
\hline
\end{tabular}




\subsection{Pulsed Water Tests}

Effects of pulsed water were investigated Figure 3-6. Different water flow rate was used. The pulse cycle was 2 seconds and duty was $50 \%$.

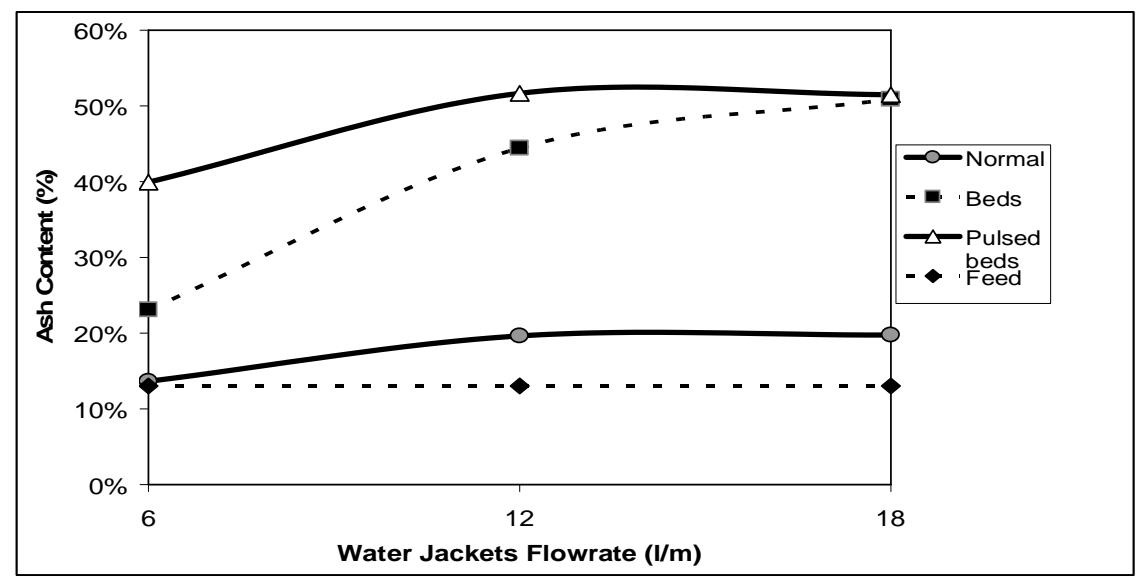

Figure 3-6. Effects of Pulsed Artificial beds on the Concentrate Grade

It can be seen from Figure 3-6, that artificial beds can significantly improve the separation efficiency. At a flow rate of $18 \mathrm{1} / \mathrm{min}$ the ash content was increased from less than $20 \%$ to more than $40 \%$. Combined with pulsation, the separation performance can be further improved even at lower flow rates of 6 and $12 \mathrm{l} / \mathrm{min}$. At a flow rate of $18 \mathrm{l} / \mathrm{min}$, pulsation combined an artificial bed did not significantly improve the ash content of the concentrate compared with that of artificial bed only. This observation was similar to the results produced when pulsation alone was used.The above figure shows that the artificial beds and pulsed artificial beds can significantly improve the concentration grade, nearly double the grade. Using pulsed water with beds can improve even more the grade, especially at the low water flow rate.

From the above experiments, the effect of the pulsed water alone can not be shown. Another set of experiments about the effect of the pulsed water alone was conducted. The same pulsed water was used. The results are shown in Figure 3-7.

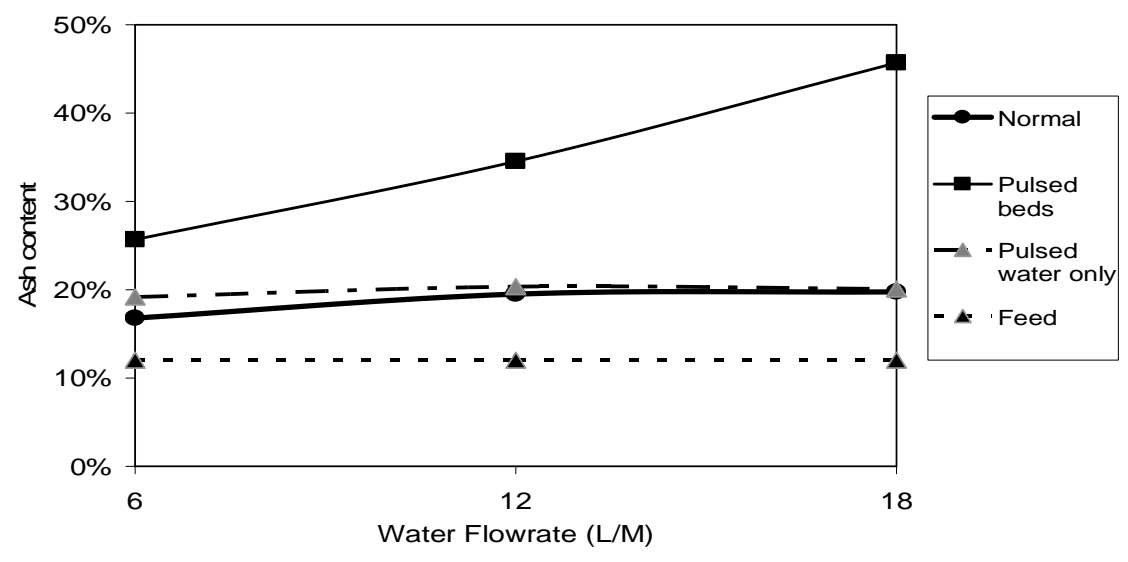




\section{Figure 3-7. Effects of Pulsed Water on the Concentrate}

It indicates the pulsed water can improve the concentrate grade. Pulsed water without beds slightly can improve the concentrate grade at low water flow rate. Pulsed water with beds can significantly improve the concentrate grade. The artificial beds have more positive effects on the concentrate grade Figure 3-8.

Different frequencies have different effects on the jigging separation. Typical jigging cycles are from 1 second to 3 seconds [20]. Another cycle period of 1 second of pulsed water was used to investigate the effect of the pulsed water on the concentrate grade.

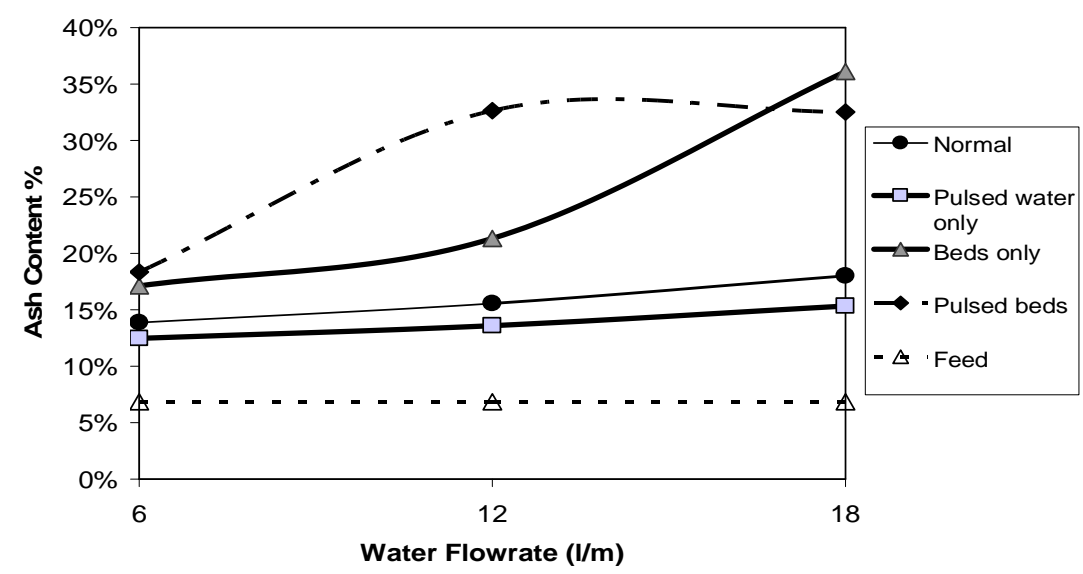

Figure 3-8. Effects of Pulsed Water on the Concentrate Grade

It can be concluded from Table 2 that artificial porous beds can significantly improve the separation performance of the Knelson concentrator. Use of pulsed fluidisation water at the appropriate frequency can improve the separation performance at lower flow rates. In the table pulsation was shown the improvement ratio increased about 1.25 compared with artificial bed only. In the other hand, lower cycle period pulsed water alone has a negative effect on the concentrate grade. But with artificial beds, it still can improve the concentrate grade at lower flow rate.

Table 2. Ash contents with, with out bed, with and without pulsation in product

\begin{tabular}{|l|r|r|r|}
\hline Flow rate & 6 & 12 & 18 \\
\hline Normal & $13.87 \%$ & $15.56 \%$ & $18.01 \%$ \\
\hline Pulsed water only & $12.47 \%$ & $13.59 \%$ & $15.33 \%$ \\
\hline Beds only & $17.12 \%$ & $21.32 \%$ & $36.11 \%$ \\
\hline Pulsed Beds & $18.35 \%$ & $32.64 \%$ & $32.52 \%$ \\
\hline Feed & $6.85 \%$ & $6.85 \%$ & $6.85 \%$ \\
\hline Improvement Ratio with pulsed water only & -0.101 & -0.127 & -0.149 \\
\hline Improvement Ratio with bed only & 0.2343 & 0.3702 & 1.005 \\
\hline Improvement Ratio with pulsed bed & 0.3593 & 1.2568 & 0.9465 \\
\hline
\end{tabular}




\section{Modelling of the Separation Mechanism}

Fluidization and enhanced gravitational force is the main principal. The enhanced gravitational force is achieved by the centrifugal movement. The fluidization water is introduced at the base of the ring to keep the particle bed in suspension. This allows the terminal velocity of the settle particles to be kept in balance with the inward water flow to allow the separation of particles [21]. Although this kind of single particle terminal velocity model has gained somewhat success for the binary solids differing only in density with artificial beds [19], it is not perfect for the binary solids fluidized bed system.

\subsection{Knelson Concentrator Mechanisms}

As stated previous, case studies pertaining to particular applications of Knelson concentrators have been well documented, but descriptions of the mechanism involved or the theoretical considerations have been limited. In order to better understand the segregation mechanism in a centrifugal fluidised bed, the particle behaviour and velocity in the bed have to be determined. The velocity equations for a particle in a centrifugal fluidised bed and macroscopic conservation equations are presented in this section.

\subsubsection{Free Settling Velocity}

In a centrifugal fluidised bed, particles are fluidised in a centrifugal field. The bed usually consists of a cylindrical basket which rotates about its axis of symmetry. The rotation about the axis causes the particles in the basket to form an annular region at its circumference. Fluid is injected inward through the perforated surface of the basket wall and the particles begin to fluidise when the force exerted by the fluidising medium becomes equal to the effective weight of particles in the centrifugal field Figure 4-1.

A situation where a fluid rotates as a rigid body and flows axially parallel to the rotation axis in a laminar fashion has also been considered [22]. As all particles are assumed to be very small in size,

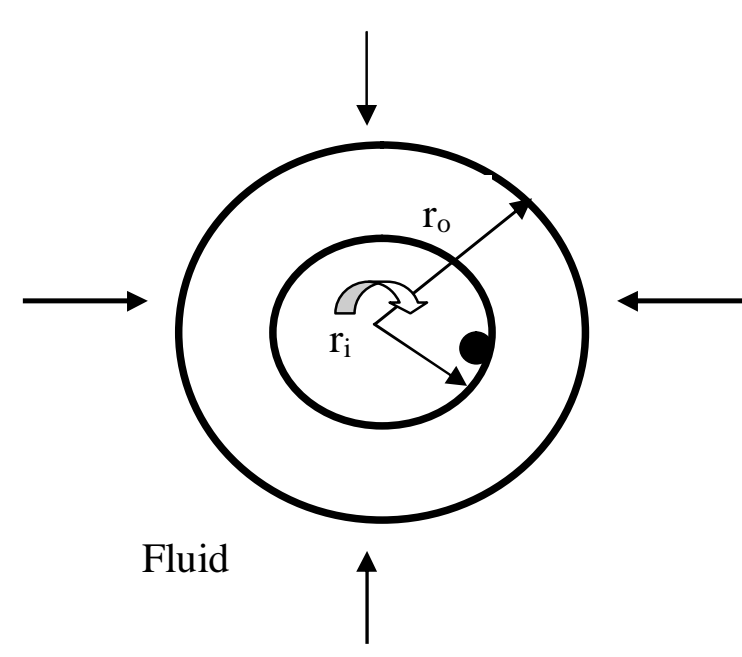
Figure 4-1. Schematic of a centrifugal particle bed the inertial forces acting on them are small. Particle concentrations are small so that interactions can be ignored. Particles follow the streamlines of the fluid except for the radial direction where as result of centrifugal and buoyancy forces they move relative to the fluid [22]. The radial velocity of a particle moving through a liquid under the influence of a centrifugal force field is given by a combination of the centrifugal force $F_{c}$, buoyancy $F_{b}$, and drag force $F_{d}$ [23];[22].

For creeping flow, the drag force $F_{d}$ exerted on a single particle can be described by Stokes' law:

$$
F_{d}=3 \pi \iota_{f} d_{p} v_{p}
$$


In a centrifugal field, the centrifugal force $F_{c}$ and buoyancy $F_{b}$ are given as follows, including particle density, fluid density, angular velocity, and radial coordinate $r$

$$
\begin{aligned}
& F_{c}=\frac{\pi}{6} \rho_{p} d_{p}^{3} \omega^{2} r \\
& F_{b}=\frac{\pi}{6} \rho_{f} d_{p}^{3} \omega^{2} r
\end{aligned}
$$

Using Newton's second law the following second-order difference equation is obtained to describe the particle movement:

$$
\frac{\pi}{6}\left(\rho_{p}-\rho_{f}\right) d_{p}^{3} \omega^{2} r-3 \pi \iota_{f} d_{p} \frac{d r}{d t}=\frac{\pi}{6} d_{p}^{3} \rho_{p} \frac{d^{2} r}{d t^{2}}
$$

Assuming that the time scale in which the final particle velocity reached is short compared to the overall process, and thus $r$ can be considered to be a constant. The Equation 4.1.4 can be solved by integration [23]. The radial particle free settling terminal velocity $v_{t}$ is given as the following:

$$
v_{t}=\frac{\left(\rho_{p}-\rho_{f}\right) d_{p}^{2} \omega^{2} r}{18 \mu_{f}}
$$

\subsection{Binary Solids Differing Only in Size or Density}

Actually below the single particle terminal velocity, a fluidized bed can be created. In the centrifugal field, the closer to the axis of spinning bowl, the less outward centrifugal force on the particles. The inward drag force may take advantage, and because of the limit of the depth of ring, the fluidized particles may be flowed out of the ring and be spun out as overflow. In the fluidized bed, the superficial velocity needed to support a bed of particles is much less than the terminal velocity for individual particles [24]. Therefore, the separation velocity is between the minimum fluidization velocity of larger or denser particles and the terminal velocity of smaller or lighter particles.

Liquid fluidization of binary mixture of particles differing only in size of density generates a two layer bed, with larger or denser particles settling at the bottom and the smaller or lighter at the top. If the particles sizes or densities are sufficiently different, then the two layers will be sharply separated [10].

At low Reynolds numbers, the terminal velocity can be determined by Stokes' law [24].

$$
u_{t}=\frac{g d_{p}^{2}\left(\rho_{p}-\rho_{f}\right)}{18 \mu}
$$

The Ergun equation can be used to predict the minimum fluidization velocity [24].

When the $R_{e p}$ is below 10, a simplified form of Ergun equation, the Blake-Kozeny equation, can be used to calculate the small particles minimum fluidization velocity.

$$
\frac{150}{R_{e p}}=\frac{d_{p}\left(\rho_{p}-\rho_{f}\right) g \varepsilon^{3}}{u_{m}^{2} \rho_{f}}
$$

Where in the porous particle bed

$$
R_{e p}=\frac{\rho_{f} u_{m} d_{p}}{\mu(1-\varepsilon)}
$$


In centrifugal fluidization bed, the gravitational acceleration $g$ should be replaced by the centrifugal acceleration $r \omega^{2}$ [25]; [26]. If the $\mathrm{G}$ is defined as $G=r \omega^{2} / g$, Equation 4.2 can be rewritten to:

$$
u_{m}=\frac{\left(\rho_{p}-\rho_{f}\right) g d_{p}^{2} \varepsilon^{3}}{150 \mu(1-\varepsilon)} G
$$

Thus, the minimum fluidization velocity can be obtained

\subsection{Binary Solids Differing In Size and Density}

For binary particles differing in size and density such that the larger particles are lighter and the smaller particles are denser, the segregation pattern is more sophisticated and layer inversion will appear. This phenomenon has been described previously.

The critical role of bulk density in determining the segregation and inversion behaviour of multi-component fluidized system has been acknowledged [14]. And another factor is the local voidage fluctuations which make the liquid fluidization non-uniform [7].

The bulk density of each segregated layer of particles known as

$$
\begin{aligned}
& \rho_{b 1}=\varepsilon_{1} \rho_{f}+\left(1-\varepsilon_{1}\right) \rho_{p 1} \\
& \rho_{b 2}=\varepsilon_{2} \rho_{f}+\left(1-\varepsilon_{2}\right) \rho_{p 2}
\end{aligned}
$$

At the inversion velocity the bulk densities of two layers are equal,

$$
\rho_{b 1}=\rho_{b 2}
$$

In the fluidization process, the voidage can be calculated by using a force balance [7]. This model can set up a relation between the voidage and liquid velocity for sphere particles. In both a mono-component or in a binary mixture fluidized bed, the solid particles are always balanced by the gravity, buoyancy and drag force.

The gravity

$$
F_{g}=\frac{\pi}{6} d_{p}^{3} \rho_{p} g
$$

The buoyancy

$$
F_{b}=\frac{\pi}{6} d_{p}^{3} \rho_{b} g
$$

Here $\rho_{b}$ is the bulk density.

$$
\rho_{b}=\varepsilon \rho_{f}+(1-\varepsilon) \rho_{p}
$$

The drag force

$$
F_{d}=C_{d} \frac{\pi}{8} d_{p}^{2} \rho_{f} u^{2} \varepsilon^{-4.7}
$$

When the liquid fluidized bed attains the equilibrium condition at given liquid velocity. The corresponding force balance is achieved. The voidage can be determined by combining the equations (4.3.4), (4.3.5), (4.3.6) and (4.3.7)

$$
\varepsilon=\left(\frac{4 d_{p}\left(\rho_{p}-\rho_{f}\right) g}{3 C_{D} \rho_{f} u^{2}}\right)^{-1 / 5.7}
$$

With the centrifugal force, the equation 4.3 .8 can be re-written as: 


$$
\varepsilon=\left(\frac{4 d_{p}\left(\rho_{p}-\rho_{f}\right) g}{3 C_{D} \rho_{f} u^{2}} G\right)^{-1 / 5.7}
$$

The voidages of two layers of particles can be computed from the equation (4.3.2)

$$
\begin{aligned}
& \varepsilon_{1}=\left(\frac{4 d_{p 1}\left(\rho_{p 1}-\rho_{f}\right) g}{3 C_{D 1} \rho_{f} u^{2}} G\right)^{-1 / 5.7} \\
& \varepsilon_{2}=\left(\frac{4 d_{p 2}\left(\rho_{p 2}-\rho_{f}\right) g}{3 C_{D 2} \rho_{f} u^{2}} G\right)^{-1 / 5.7}
\end{aligned}
$$

The inversion velocity can be obtained by substitution of Equation (4.3.1), (4.3.2), (4.3.10) and (4.3.11) into Equation (4.3.3).

\section{Conclusions}

The effects of artificial porous beds and pulsed fluidisation water have been investigated. This study showed that artificial beds can significantly improve the separation performance of the Knelson concentrator. Pulsed fluidisation water can improve the performance with and without artificial beds, especially for low flow rates.

It was found that creating an artificial porous bed in the rings of the concentration bowl could significant improve the grade of the concentrate. The improvement ratio was achieved is about 1.55 compared with no bed for the same flow rate.

Using pulsed water with beds can improve the grade as well; the improvement ratio achieved in this case was about 1.25 . The shorter cycle period pulsed water alone has a negative effect on the concentrate grade. But with artificial beds, it still can improve the concentrate grade at lower flow rate.

Two mathematic models have been developed. In these two models, the separation velocity that is related to the water flow rate or the enhanced gravity force that is related to the bowl speed can be determined.

\section{References}

1. Knelson Concentrators Inc. (2003) "Knelson kc-cvd6 concentrator installation, operation and maintenance manual". knelson gravity solution. Canada.[on line] available at www.knelson.com.

2. Rand, P.; Miles, N.J.; Large, D.J.; Rice, G.E. ( 1999 ) "Remediating Contaminated Canal Sediments and Sewage Sludge Incinerator Ashes Using High-g Gravity Separation" Proceedings of the TMS Fall Extraction and Processing Conference, v 3, p 2551-2560.

3. Galbraith, P. Devereux, J. (2002) "Beneficiation of Printed Wiring Boards with Gravity Concentration". IEEE International Symposium on Electronics and the Environment, 242248.

4. McLeavy, M. Klein, B. and Grewal, I. (2001) "Knelson Continuous Variable Discharge Concentrator: Analysis of Operation Variables". International Heavy Minerals Conference.

5. Deer2 (2002) 2Final CDRL C005 Equipment Upgrade Report MNS Subtask ME-07 PWB Recovery Separation Process-Knelson Concentrator" [on line] Available at:< http://www.deer2.com/metals/pwb_recovery_knelson_concentrator.pdf> .

6. Kunii, D., Levenspiel, O., (1991) "Fluidization Engineering" Butterworth-Hernmann, Boston.

7. $\mathrm{Hu}, \mathrm{X}$., (2002) "The segregation in the binary-solid liquid fluidized bed" Chemical Engineering Technology 25 911-915. 
8. Rasul, M., Rudolph, V., Wang F. (2000) Particles separation using fluidization techniques. Int. J. Miner. Process. 60 163-179.

9. Moritomi, H., Yamagishi, T., Chiba, T., (1986) "Prediction of complete mixing of liquidfluidized binary solid particles" Chemical Engineering Science. 41 297-305.

10. Felice, R., (1995) "Hydrodynamics of liquid fluidization" Chemical engineering science 50 (8) 1213-1245.

11. Jean, R. H., Fan, L. S. (1986) "On the criteria of solids layer inversion in a liquid-solid fluidized bed containing a binary mixture of particles" Chemical Engineering Science 41 2811-2821.

12. Patwardhan, V. S. and Chi Tien, (1985b) "Sedimentation and liquid fluidization of solid particles of different sizes and densities" Chemical Engineering Science. 40 1051-1060.

13. Moritomi, H., Iwase, T., Chiba, T., (1982) "A comprehensive interpretation of solid layer inversion in liquid fluidized beds" Chem. Eng. Sci. 37 (12), 1751-1757.

14. Epstein, N. LeClair, B.P. (1985) "Liquid fluidization of binary particle mixture II bed inversion" Chemical Engineering Science 401517 -526.

15. Gibilaro, L.G., di Felice, R., Waldram, S.P., Foscolo, U. P., (1986) "A predictive model for the equilibrium composition and inversion phenomena of binary-solid liquid fluidized beds" Chemical Engineering Science 41 379-387.

16. Asif, M. (1998) "Segregation Velocity Model for Fluidized Suspension of Binary Mixture of Particles" Chemical Engineering and Processing 37, 279-286.

17. Richardson, J., Zaki, W. (1954) "Sedimentation and fluidization" Trans. Inst. Chem Eng., 32 35-53.

18. Ling, J. (1998) "A study of a variable speed 3-in Knelson concentrator" $\mathrm{PhD}$ thesis, McGill University, Canada.

19. Rand, P. (2000) "An investigation of the Mode of Operation of the Knelson concentrator". PhD thesis, The University of Nottingham, UK.

20. Galvin, K. Pratten, S. Lambert, N. Callen, A. Lui, J. "Beneficiation of Coal Using a Teetered Bed Separator with Jigging Action" ACARP End of Grand Report Project No. C9049.

21. Fullam, M. Grewal, I. (2001) "The Knelson Continuous Variable Discharge (CVD) Concentrator". The Processing for Profit Conference in Atlanta, Georgia.

22. Brouwers, J. J. H. (2002) "Phase separation in centrifugal fields with emphasis on the rotational particle separator". Experimental Thermal and Fluid Science, 26, 325-334.

23. Biesheuvel, P. M., Nijmeijer, A. and Verweij, H. (1998) "Theory of batchwise centrifugal casting". AIChE Journal, 44, 1914-1922.

24. McCabe, W. Smith, J. Harriott, P. (2001). "Unit Operation of Chemical Engineering" $6^{\text {th }}$ edition. McGraw-Hill Higher Education P172.

25. Chen, Y. (1987) "Fundamentals of a Centrifugal Fluidized Bed" AIChE Journal 33 722728.

26. Kao, J. Pfeffer, R. Tardos, G. (1987) "On Partial Fluidization in Rotating Fluidized Beds". AIChE Journal 33 858-861. 